\title{
Internalized stigma of mental illness in Sweden and Iran- A comparative study
}

\author{
Lars Jacobsson $^{1 *}$, Helia Ghanean ${ }^{1,2}$, Birgitta Törnkvist ${ }^{3}$ \\ ${ }^{1}$ Department of Psychiatry, Umeå University, Umeå, Sweden \\ ${ }^{2}$ Tehran Medical University, Tehran, Iran \\ ${ }^{3}$ Department of Statistics, Umeå University, Umeå, Sweden \\ Email: lars.jacobsson@psychiat.umu.se
}

Received 4 June 2013; revised 5 July 2013; accepted 12 July 2013

Copyright (c) 2013 Lars Jacobsson et al. This is an open access article distributed under the Creative Commons Attribution License, which permits unrestricted use, distribution, and reproduction in any medium, provided the original work is properly cited.

\begin{abstract}
Objectives: Stigma and discrimination because of mental illness is still prevalent even in high-income countries where a lot of emphasis has been given to anti-stigma activities and measures to reduce the marginalization of these persons. One aspect of the stigma issue is the self-stigmatization that persons suffering from mental illness are experiencing. In this study two different societies have been compared as regards internalized stigma of mental illness. Methods: A widely used questionnaire was used by the Internalized Stigma of Mental Illness scale (ISMI). One sample of psychiatric patients from Sweden $(\mathrm{N}=$ 163) and from Iran $(N=138)$ is compared. Results: The Swedish sample generally reports lower levels of experienced stigma except for items covering selfblame and feelings of alienation. Conclusions: There are reasons to consider the aspect of self-stigmatization when working with mentally ill persons even in high income countries with well developed mental health services.
\end{abstract}

Keywords: Mental Disorders; Internalized Stigma; Iran; Sweden

\section{INTRODUCTION}

Stigma and discrimination because of medical conditions, such as epilepsy, leprosy, cancer and even HIV/AIDS has decreased over the years in most high income countries with good health care and a general public which is rather well knowledgeable in health issues. As regards mental illness the situation is not that positive. Stigma and discrimination because of mental disorders is still highly prevalent even in western high income countries

\footnotetext{
*Corresponding author.
}

[1]. A number of studies from many countries indicate that it may be a lower level of stigma because of depressive disorders, but instead several studies indicate that mental illness is considered to be dangerous to an even higher extent than earlier [1,2]. The most important consequences of this are that those persons with mental health problems do not seek psychiatric help until late in the course of their illness and that mental health services do not get resources according to the needs in the population. In Sweden, for example, the funding of research on cancer is ten times higher than what is allocated to psychiatric research even though the societal costs for cancer are only half as high as those for mental disorders [3].

One aspect of the stigma complex is the experience of stigma by persons suffering from mental disorders. The issue of self-stigmatization might be as important as that of stigmatization by the surrounding society and the literature on internalized stigma has increased over the last decade. Internalized stigma has been described by Ritcher and co-workers as "The devaluation, shame, secrecy and withdrawal triggered by applying negative stereotypes to oneself" [4]. They have developed a questionnaire, "the Internalized Stigma of Mental Illness scale (ISMI), which has now been used in a number of studies from different cultures" [4-6]. We have used this scale to study internalized stigma of mental illness in Tehran, Iran [7] and we thought it might be of interest to compare the results with data of patients in Sweden suffering from mental disorders.

One idea of this study is to compare the situation in a country with a strict adherence to Islamic values with a modern secularized western society. Medical care in Iran is well developed and the educational level of the population is also rather high. According to the Holy Koran, people with mental disorders should be treated well and with respect [8]. This might result in a more tolerant attitude. In a more secular western culture there have been 
several attempts to change the general perception of the population into a more tolerant and open attitude towards mental illness over the years through for example national anti-stigma campaigns [9].

Another perspective is the possible differences between Sweden and Iran because of the different sociocultural contexts. Sweden is an extremely individualistic society and Iran is more collectivistic.

Hansson has made an interview study on perceived and anticipated discrimination in people with mental illness in Sweden and they found quite a high level of perceived discrimination in spite of several actions over the years to reduce stigma and discrimination because of mental illness in Sweden [10].

So, the aim of this study is to compare the experience of stigma/internalized stigma in patients suffering from mental disorders in Sweden and Tehran, Iran.

\section{METHODS}

The Swedish study was conducted in Umea, a university town in northern Sweden. The Swedish sample consists of a convenience sample of persons attending the psychiatric clinic of the university hospital, which is the only psychiatric clinic in the region with a catchment area of around 200,000 inhabitants. The Swedish patients $(\mathrm{N}=163)$ were recruited from the outpatient department with a mix of diagnoses over the whole spectrum of mental disorders (53\% affective disorders). In the Swedish, sample $67 \%$ were female and the majority was middle aged (around 35 years of age). $42 \%$ had spent more than 12 years at school. The Iranian sample $(\mathrm{N}=138)$ was recruited from out-patients and in-patients at two different psychiatric clinics in Tehran. The Iranian sample and the results are described more in detail separately (7). $60 \%$ were males, the mean age 30 years and $72 \%$ had high school diploma or higher education (12 years and more).The majority had an affective disorder (67\%).

The Internalized Stigma of Mental Illness scale (ISMI) was used in both settings. It contains 29 items grouped into five subscales; "Alienation", "Stereotype endorsement”, "Perceived discrimination”, "Social withdrawal" and "Stigma resistance". These subscales were basically theory driven and the authors conclude that "it is most parsimonius to conceptualize the ISMI as measuring a single construct". The 29 items have to be rated on a four-point-Likert scale $(1=$ strongly disagree to $4=$ strongly agree), which implies that high scores reflect more severe stigmatization for all items except for the Stigma resistance scale where the items are reversely coded. Participation in both studies was voluntary and conducted according to the principles of the Helsinki declaration. The two studies were approved by relevant research ethics committees in Tehran and Umea.

The IBM/SPSS package version 18 was used to calcu- late per cents agreeing to the questions in Sweden and Iran, mean and standard deviation of the subscales and total scale, Cronbachs alpha for the subscales and total scale, and midpoint and stigma level in Sweden. Microsoft Office Excel 2007 was used to make the figure of the differences between Sweden and Iran.

\section{RESULTS}

In Figure $\mathbf{1}$ is shown a comparison on item-level between the two settings. In the figure are the two response alternatives 3 and 4 aggregated ("agree" and "strongly agree") into an "agree"-response. The differences are significant at $5 \%$ level if the difference is $10.5 \%$ or more between the two samples. Generally the Teheran sample indicates a higher level of experienced stigma than the Swedish sample. However, the Swedish sample differs significantly on some items belonging to the subscale "Alienation" scoring higher than the Teheran sample.

The highest difference between the proportion of persons that agreed to the statements was found for item 7 . (People with mental illness can make important contributions to society.) The difference between the percent of persons in the Swedish sample that agreed to item 7 and percent in Tehran that agreed is $40 \%$. Ninety percent in the Swedish sample agreed to item 7 compared to 50\% in the Tehran sample.

In Table $\mathbf{1}$ are presented some data on subscale level. Cronbachs Alfa for the two samples is acceptable except for the Stigma resistance scale as regards the Swedish sample. The mean scores are in line with the findings on item level. Items belonging to the subscales Stereotype endorsement (6 out of 7 items) and Discrimination experience (4 out of 5) differ most from the Swedish group. The questions that differed most was item 19 "Because I have a mental illness, I need others to make most decisions for me", item 3 "People discriminate against me because I have mental illness", item 15 "People often patronize me or treat me like a child because I have a mental illness", and item 6 "Mentally ill people should not get married". Items 19 and 6 belong to the subscale "Stereotype endorsement" and item 3 and 15 to "Discrimination experience".

The Swedish sample differs on items belonging to the subscales "Stigma resistance" (items 7, 27, 26) and "Alienation" (items 5, 16, 8 and 1). Item 7 goes "People with mental illness can make important contributions to society", item 27 "I can have a good fulfilling life despite my mental illness" and item 26 "In general, I am able to live my life the way I want to". The Swedish group endorsed more often items belonging to the alienation subscale as item 5 "I am embarrassed or ashamed that I have a mental illness" and item 16 "I am disappointed in myself for having a mental illness”.

In the Swedish sample almost half of the respondents 


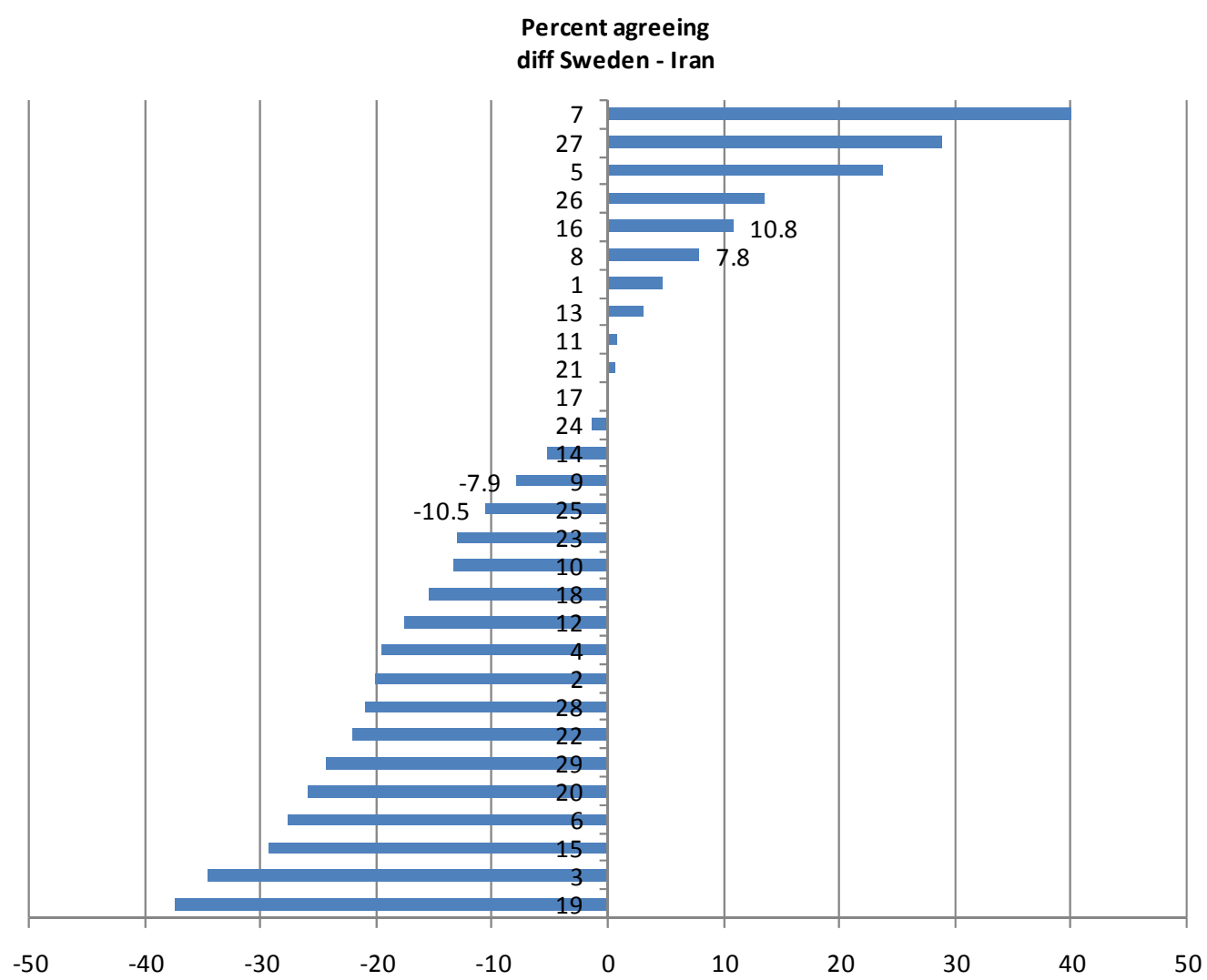

Figure 1. Item comparisons between Sweden and Iran, differences in per cent agreeing to the questions.

Table 1. Cronbachs alpha and mean (SD) for the subscales and the total scale for Sweden $(\mathrm{N}=188)$ and $\operatorname{Iran}^{*}(\mathrm{~N}=138)$, respectively.

\begin{tabular}{lcccc}
\hline Scale & Cronbachs alpha Sweden & Cronbachs alpha Iran & Mean (SD) Sweden & Mean (SD) Iran \\
\hline Alienation & 0.80 & 0.81 & $2.57(0.71)$ & $2.33(0.73)$ \\
Stereotype endorsement & 0.70 & 0.77 & $1.61(0.48)$ & $2.30(0.60)$ \\
Percieved discrimination & 0.81 & 0.80 & $1.81(0.68)$ & $2.32(0.67)$ \\
Social withdrawal & 0.79 & 0.77 & $2.08(0.68)$ & $2.64(0.64)$ \\
Stigma resistance & 0.55 & 0.89 & $2.26(0.57)$ & $2.46(0.39)$ \\
Total & 0.90 & & $2.05(0.48)$ & \\
\hline
\end{tabular}

*From the paper by Ghanean et al. (7).

had made comments. The majority of the comments in the Swedish sample deal with negative experiences fitting well into the concepts of the five proposed subscales. There were quite a number of comments on the psychiatric care, both negative and positive. The negative dealt with feelings of being treated in a derogating way by staff, not listened to, lack of continuity as regards doctors and nurses, and only getting drugs instead of psychotherapy. However there were also positive experiences of "being seen", "being well-treated" and "getting a lot of support”.

Quite a few $(\mathrm{N}=11)$ reported that they were not taken seriously by other doctors when they were referred from the psychiatric clinics for somatic problems. Quite a few also mentioned problems in getting jobs or losing jobs because of their mental problems. There were notes that they tried to hide their problems for their family and workmates and feelings of abandonment. Interestingly no one mentioned anything about the idea that mentally ill persons are dangerous and aggressive, which was a rather common comment in the Iranian sample.

In the Iranian sample almost all participants $(\mathrm{N}=123)$ responded to the open-ended question. The responses indicated feelings of alienation and discrimination; "There is no cultural understanding in our society; they ridicule us", "If my family realized that I am sick they 
would abandon me because they think it is in the genes". A recurring theme was the idea that mentally ill persons are dangerous; "Neighbours are scared of us", "They all believe that a mentally ill person is a natural born killer".

\section{DISCUSSION}

There are several limitations of this study. The two samples are both convenience samples and it is difficult to tell how representative they are for mentally ill persons in general in the two settings. In the Iranian sample a majority had an affective disorder and in the Swedish sample there was a somewhat broader mix of diagnostic categories. The Iranian patients seemed to have a higher educational level and were somewhat younger than the Swedish sample. It is difficult to say what these differences in the two samples might mean, but our feeling is that the two samples still reflect a rather representative group of persons suffering from mental illness of clinical significance in both settings.

As regards the level of stigma it is difficult to be explicit as there are no norms for what is a high level or a low level of stigma. The group that developed ISMI [4] suggests to use the midpoint of the scale (an average score equal or above 2.5 on the 1 - 4 point scale) as a possible level of high internalized stigma. In Table 2 this is illuminated indicating that $16 \%$ of the Swedish group experience high stigma compared to $40 \%$ of the Teheran sample. Another way of determining the level of stigma is suggested by Lysaker et al. [11]. They propose that a score of 2 or less should be labelled "minimal stigma", scores between 2 and less than 2.5 could be labelled as "mild stigma", scores between 2.5 and 3 could be labelled "moderate stigma" and scores greater than 3 could be labelled as "severe stigma". If we use this model the results are shown in Table 2 . Here are also data from two studies using ISMI in 14 European countries on people with schizophrenia [5] and affective disorders [12]. Even here it is obvious that the Swedish sample report a lower level of moderate and severe stigma according to this model.

If we look at the item level, the Swedish sample score lower than the Iranian in seventeen items of the scale, indicating a lower level of stigma. However, in seven items the Swedish sample score higher than the Iranian as is shown in Figure 1.

Almost all in the Iranian sample responded to the open-ended question and gave a lot of examples of experiences and feelings of marginalization and discrimination. Only around $40 \%$ of the Swedish sample made comments and quite a few $(\mathrm{N}=31)$ dealt with the medical care they have got. One third of these who responded were critical to the treatment they got from the psychiatric staff, but another third also expressed their satisfaction with the staff. Several told that they were not taken seriously by other doctors when attending because of somatic problems. It seems as these Swedish patients are more observant on the way they are treated by psychiatric and other medical staff compared to what is expressed in the Iranian group. This might be a consequence of the strong emphasis, which is now put on patients' rights in the Swedish society.

The results are interesting from several points of view. Generally it seems as if the level of experienced stigma is higher in the Iranian sample than in the Swedish, but there is a paradoxical pattern in that the Swedish sample indicates a higher level of experienced stigma when it comes to self-blame and feelings of alienation. At the same time the Swedish sample score higher on some of the items in the resistance scale, for example "In general I am able to live my life the way I want” (item 26) and "I can have a good, fulfilling life in spite of my mental illness" (item 27) indicating a more favourable situation. So, the Swedish patients indicate a lower level of what might be seen as external stigmatization and the same and maybe even higher levels of self blame and shame than the Iranian patients. This might be a consequence of the emphasis on the role of the individual person and his/her responsibility in the Swedish society. When comparing the levels of stigma with the figures from the big European multicenter studies it seems that both the Iranian and the Swedish sample indicate a lower level of stigmatization than the European samples. However, one

Table 2. Comparison of stigma levels (in per cent) according to ISMI in Sweden, Iran and Europe.

\begin{tabular}{ccccc}
\hline Midpoint & Sweden (N = 188) & Tehran $(\mathbf{N}=\mathbf{1 3 8})$ & Europe* $^{*}$ (Schiz N = 1211) & Europe $^{* * *}$ (Affect N = 1182) \\
\hline$<2.5$ & 84 & 60 & 57 & 78 \\
$>2.5$ & 16 & 40 & 43 & 22 \\
Stigma level & & & & \\
Minimal $(<2)$ & 47.2 & 40 & 23 & 45.6 \\
Mild (2 - 2.49) & 36.8 & 21 & 34 & 30.8 \\
Moderate (2.5 - 3) & 11.7 & 27 & 29.4 & 18.1 \\
Severe (>3) & 4.3 & 12 & 12.5 & 3.6 \\
\hline
\end{tabular}

"Brohan et al. (5), ** Brohan et al. (12). 
of the European samples consists of patients with schizophrenia and this probably explains part of the difference. Patients suffering from schizophrenia are probably the most stigmatized group of psychiatric patients and the number of patients with this diagnose is lower in the Swedish and the Iranian sample.

\section{CONCLUSION}

It seems that if the Swedish patients experience a lower level of stigma than the sample from Iran, and it's concluded upon comparing to the two big European multicenter studies. The studies are not directly comparable because of differences in diagnostic groups, but on a more global level we think it is possible to draw some conclusions. The possible lower level of experienced stigma in the Swedish sample might be a consequence of the efforts in the Swedish society over the years to reduce stigma and discrimination of mentally ill persons. However, it is somewhat paradoxical to see that the Swedish sample expresses higher levels of self-blame and feelings of alienation than the Iranian sample. So, one possibility is that there is less external stigmatization in the Swedish sample, but still high levels of selfblame and shame. This should be addressed in the Swedish care setting.

\section{REFERENCES}

[1] Angermeyer, M.C. and Dietrich, S. (2006) Public beliefs about and attitudes towards people with mental illness: A review of population studies. Acta Psychiatrica Scandinavica, 113, 163-179.

http://dx.doi.org/10.1111/j.1600-0447.2005.00699.x

[2] Ineland, L., Jacobsson, L., Salander, R.E. and Sjölander, P. (2008) Attitudes towards mental disorders and psychiatric treatment-Changes over time in a Swedish population. Nordic Journal of Psychiatry, 62, 192-197. http://dx.doi.org/10.1080/08039480801962855

[3] The Royal Swedish Academy of Sciences (2011) Reformprogram för svensk forskning.
[4] Ritsher, J.B., Otilingam, P.G. and Grajales, M. (2003) Internalized stigma of mental illness: Psychometric properties of a new measure. Psychiatry Research, 121, 31-49. http://dx.doi.org/10.1016/j.psychres.2003.08.008

[5] Brohan, E., Elgie, R., Sartorius, N. and Thornicroft, G. (2010) Self-stigma, empowerment and perceived discrimination among people with schizophrenia in 14 European countries: The GAMIAN-Europe study. Schizophrenia Research, 122, 232-238.

http://dx.doi.org/10.1016/j.schres.2010.02.1065

[6] Assefa, D., Shibre, T., Asher, L. and Fekadu, A. (2012) Internalized stigma among patients with schizophrenia in Ethiopia: A cross-sectional facility-based study. Psychiatry, 12, 239.

[7] Ghanean, H., Nojomi, M. and Jacobsson, L. (2011) Internalized stigma of mental illness in Tehran, Iran. Stigma Research and Action, 1, 11-17. http://dx.doi.org/10.5463/sra.v1i1.10

[8] Bolhari, J., Nouri Ghassem Abadi, R. and Ramezani Farani, A. (2002) Hussain Khan, Z., Ed., Quranic verses mental health for mental health workers and school counsellors (p. 22). Office for Islamic Studies of Mental Health, Tehran.

[9] Ritsch, N., Angermeyer, M.C. and Corrigan, P.W. (2005) Mental illness stigma: Concepts, consequences, and initiatives to reduce stigma. European Psychiatry, 20, 529539. http://dx.doi.org/10.1016/j.eurpsy.2005.04.004

[10] Hansson, L. (2013) Perceived and anticipated discrimination in people with mental illness. An interview study. Nordic Journal of Psychiatry, in press. http://dx.doi.org/10.3109/08039488.2013.775339

[11] Lysaker, P.H., Davis, L.W., Warman, D.M., Strasburger, A. and Beattie, N. (2007) Stigma, social function and symptoms in schizophrenia and schizoaffective disorder: Associations across 6 months. Psychiatry Research, 149, 89-95. http://dx.doi.org/10.1016/j.psychres.2006.03.007

[12] Brohan, E., Gauci, D., Sartorius, N. and Thornicroft, G. (2001) Self-stigma, empowerment and perceived discrimination among people with bipolar disorder or depression in 13 European countries: The GAMIANEurope study. Journal of Affective Disorders, 129, 56-63. http://dx.doi.org/10.1016/j.jad.2010.09.001 